\title{
Generating Multi-objective Optimized Configurable Business Process Models
}

\author{
A. Jiménez-Ramírez, I. Barba, C. Del Valle \\ University of Seville \\ Dpto. Lenguajes y Sistemas Informáticos, Spain \\ Email: \{ajramirez,irenebr,carmelo\}@us.es
}

\author{
B. Weber \\ University of Innsbruck \\ Department of Computer Science, Austria \\ Email: barbara.weber@uibk.ac.at
}

\begin{abstract}
The manual specification of imperative business process (BP) models can be very complex and time-consuming, potentially leading to non-optimized models or even errors. To support process analysts in the definition of these models, a method for generating optimized configurable BP models from a constraint-based specification by considering multiple objectives is described. A constraint-based specification typically allows for several different ways of executing it leading to several enactment plans which can, however, vary greatly in respect to how well different performance objective functions can be achieved. We therefore automatically generate different plans and select the ones which fit best the objectives of the company. The generated plans are then merged into an optimized configurable BP model to support the model expert in choosing the most appropriate plan depending on the importance of each objective at configuration time.
\end{abstract}

Index Terms-Business Process Management, Business Process Modelling, Constraint Programming

\section{INTRODUCTION}

A business process (BP) consists of a set of activities which are performed in coordination in an organizational and technical environment [1], and which jointly realize a business goal. Typically, BPs are specified manually in an imperative way, which can be a very complex and time-consuming process, potentially leading to non-optimized models or even errors.

To support users in the generation of optimized business process models we suggest an automatic method which takes a constraint-based process model as a starting point. Instead of focussing on how things have to be done, they allow their users to specify what has to be done [2]. A constraint-based specification typically allows for several different ways of executing it leading to several enactment plans. Therefore, we automatically generate optimized BP enactment plans from which we select the most appropriate ones regarding some objective functions to optimize (e.g., minimization of overall completion time or cost).

For generating these plans, activities to be executed have to be selected and ordered (planning), and the resources have to be allocated involving temporal reasoning (scheduling). For planning and scheduling (P\&S) the activities in an optimized way, we propose a constraint-based approach since constraint programming $(\mathrm{CP})$ supplies a suitable framework for modelling and solving $\mathrm{P} \& S$ problems [3].

From the generated optimized BP enactment plans, only those plans which are Pareto optimized are chosen, i.e., plans that are better than all other generated plans by improving at least one objective. The domain expert then has to select the most appropriate plan depending on the importance of each objective at configuration time. To facilitate this selection we are proposing to merge them into an optimized configurable BP model which is constructed via the aggregation of the generated optimized BP enactment plans [4]. A configurable BP model [4] is a modeling artifact that captures a family of process models in an integrated manner and that allows analysts to understand what these process models share, what their differences are, and why and how these differences occur.

The current work extends our previous approach [5] by considering the optimization of multiple objective functions instead of single objectives, and by generating configurable BP models from the generated optimized plans.

As a result of our work business analysts are supported in the definition of optimized imperative BP models, and in their representation in a more flexible way, i.e., as a configurable BP model. To demonstrate the feasibility of the proposed method it is applied to a running example.

\section{Proposed Method}

In the following the proposed method is described along a running example (cf. Fig. 1).

(a) ConDec-R model: In a first stage (cf. Fig 1 (a)) a constraint-based specification is created. For the BP controlflow specification, we use an extension of the constraintbased language ConDec [2], named ConDec-R [5], which also includes reasoning about resources and parallel executions. In this way, a ConDec-R model is composed by a set of activities, a set of constraints, and the resource availabilities.

As an example, Fig. 1 (a) shows a ConDec-R model which includes an activity $\mathrm{A}$ with an estimated duration of 2 , and an estimated profit of 4 , and which requires the resource $\mathrm{R} 0$ to be executed (in a similar way activities B and C are specified). This model also includes three ConDec constraints [2]: (1) RespondedExistence $(C, A)$ which forces the execution of activity $\mathrm{A}$ if activity $\mathrm{C}$ is executed, (2) Response $(A, B)$ which forces the execution of activity $B$ after the activity $A$ is finished if $\mathrm{A}$ is executed, and (3) Existence $(B, 1)$ which forces the activity $\mathrm{B}$ to be executed at least, once. Moreover, resource availabilities are shown, i.e, there is one available resource with role R0 and one with role R1. 


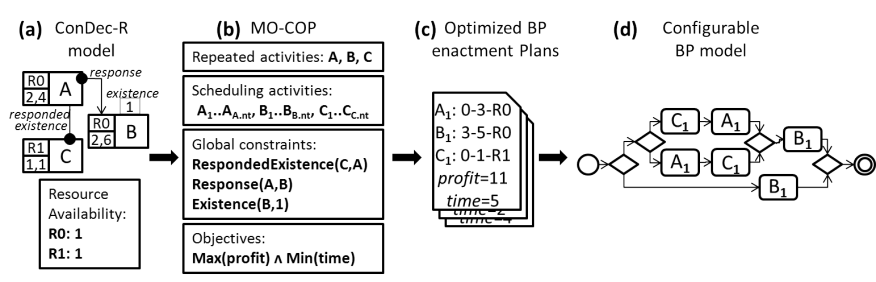

Fig. 1. Overview of our approach

(b) MO-COP: In a next step the Condec-R model is transformed into a multi-objective constraint optimization problem (MO-COP) which consists of a constraint satisfaction problem (CSP) (i.e., a set of variables, a set of domains of values for each variable, and a set of constraints which relates the variables) which also includes a set of objective functions to be optimized.

Regarding the proposed CSP model, BP activities, which can be executed arbitrarily often if not restricted by any constraints, are modelled as repeated activities, i.e, a sequence of optional scheduling activities. This is required since each execution of a BP activity needs to be allocated to a specific resource and temporarily placed in the enactment plan (for details cf. [5]). In the CSP related to the ConDec-R model of Fig. 1 (a) there are 3 repeated activities, i.e., A, $\mathrm{B}$, and $\mathrm{C}$, and the corresponding scheduling activities, i.e., $A_{1} . . A_{A . n t}, B_{1} . . B_{B . n t}, C_{1} . . C_{C . n t}$ (cf. Fig. 1 (b)), where $n t$ is the number of repetitions of a repeated activity.

To improve the modelling of the problems and to efficiently handle the constraints in the search for solutions, our constraint-based proposal includes for each ConDec template a related global constraint implemented through a filtering rule (cf. [5]), i.e., responsible for removing values which do not belong to any solution (e.g., RespondedExistence(C,A), Response(A,B), and Existence(B,1) in Fig. 1 (b)).

Since actual problems typically involve multiple conflicting objectives functions, in the current work, we built upon [5] by considering MO-COP. For the example of Fig. 1, two objective functions are declared: (1) maximization of the total profit, and (2) minimization of the overall completion time for a specific number of instances (cf. Fig. 1 (b)).

A solution to a CSP consists of assigning values to all the CSP variables, being feasible when the assignments satisfy all the constraints. In case no solution can be found (i.e., there is no variable-value assignment which satisfies all the constraints), the process analyst is informed that the constraintbased model has to be changed.

(c) Optimized BP Enactment Plans: In MO-COPs, usually no unique optimal solution exists since each solution presents specific values for different objective functions. For solving the MO-COP, we implemented a multi-objective optimization search algorithm based on the $\varepsilon$-constraint method [6], i.e., optimizing one of the objective functions constraining the other ones ${ }^{1}$. Each solution of the MO-COP corresponds to an optimized BP enactment plan. Multiple optimized BP enactment plans are then generated, but only those plans which are Pareto optimized (cf. Sect. I) are considered. In Fig. 1 (c),

\footnotetext{
${ }^{1}$ The developed algorithms were integrated with the system COMET [7].
}

the set of optimized enactment plans which are generated from the MO-COP of Fig. 1 (b) is depicted. These plans contain the following information about each executed activity: the name of the scheduling activity, its start and end times together with the resource in which is allocated (e.g., the first execution of activity A starts at time 0 , ends at time 3 , and is performed by $\mathrm{R} 0$ ). In addition, the values for the objective functions which are achieved in the plan are also depicted.

(d) Configurable BP Model: The best generated optimized BP enactment plans (i.e., those which are Pareto optimized) are then merged into an optimized configurable BP model to get a global representation which supports all the source enactment plans. The implementation of this step is based on an adaptation of the tool ProcessMerger [8] which analyzes the similarities between plans. As shown in Fig. 1(d) a configurable BP model in which each gateway is configurable is generated. A configurable gateway represents a design choice that needs to be made by an analyst at configuration time to select the most appropriate variant (i.e., optimized BP enactment plan) according to the business needs. In this way, in the configuration step, the expert can then derive a variant by configuring the model to the specific context. This variant can then be executed by a BP engine.

\section{CONCLUSION AND FUtURE WORK}

The manual specification of BP models can be complex and time-consuming, potentially leading to non-optimized models or even errors. To support process analysts in the definition of these models, this paper presents a method for generating optimized configurable BP models from constraintbased specifications. The proposed method has been fully implemented and initial experimental results are promising. As future work we plan to conduct several case studies to illustrate the feasibility of the method, and different multiobjective optimization algorithms will be analysed.

\section{ACKNOWLEDGMENTS}

This work has been partially funded by the Spanish Ministerio de Ciencia e Innovación (TIN2009-13714) and the European Regional Development Fund (ERDF/FEDER).

\section{REFERENCES}

[1] M. Weske, Business Process Management: Concepts, Languages, Architectures. Springer, 2007.

[2] M. Pesic, "Constraint-Based Workflow Management Systems: Shifting Control to Users," Ph.D. dissertation, Eindhoven University of Technology, Eindhoven, 2008.

[3] M. Salido, "Introduction to planning, scheduling and constraint satisfaction," J Intell Manuf, vol. 21, no. 1, pp. 1-4, 2010.

[4] M. Rosemann and W. van der Aalst, "A configurable reference modelling language," Inform Syst.

[5] I. Barba and C. Del Valle, "A Constraint-based Approach for Planning and Scheduling Repeated Activities," in Proc. COPLAS, 2011, pp. 55-62.

[6] Y. Haimes, L. Lasdon, and D. Wismer, "On a bicriterion formulation of the problems of integrated system identification and system optimization." IEEE Trans on Syst Man Cybern, vol. 1, pp. 296-297, 1971.

[7] Dynadec, "Comet Downloads," http://dynadec.com/support/downloads/, 2010, [Online; accessed 5-December-2011].

[8] M. La Rosa, M. Dumas, R. Uba, and R. Dijkman, "Merging Business Process Models," in OTM 2010, 2010, vol. 6426, pp. 96-113. 